\title{
Effect of molecular hydrogen on uterine inflammation during preterm labour
}

\author{
TOMOKO NAKANO $^{1}$, TOMOMI KOTANI $^{1}$, KENJI IMAI $^{1}$, YUKAKO IITANI $^{1}$, TAKAFUMI USHIDA $^{1}$, \\ HIROYUKI TSUDA ${ }^{2}$, HUA LI $^{3}$, AKIRA IWASE ${ }^{1}$, SHINYA TOYOKUNI ${ }^{4}$ and FUMITAKA KIKKAWA ${ }^{1}$
}

\author{
${ }^{1}$ Department of Obstetrics and Gynaecology, Nagoya University Graduate School of Medicine, Nagoya, Aichi 466-8550; \\ ${ }^{2}$ Department of Obstetrics and Gynaecology, Japanese Red Cross Nagoya Daiichi Hospital, Nagoya, Aichi 453-8511, Japan; \\ ${ }^{3}$ Department of Neurology, Yanbian University Hospital, Yanji, Jilin 133000, P.R. China; ${ }^{4}$ Department of Pathology \\ and Biological Responses, Nagoya University Graduate School of Medicine, Nagoya, Aichi 466-8550, Japan
}

Received August 31, 2017; Accepted February 14, 2018

DOI: $10.3892 /$ br.2018.1082

\begin{abstract}
Intrauterine inflammation causes preterm birth and is associated with complications in preterm neonates. Thus, strategies aimed at suppressing inflammation are expected to be effective for reducing the risk of preterm birth and associated complications. Our previous studies demonstrated that molecular hydrogen $\left(\mathrm{H}_{2}\right)$, an anti-inflammatory agent, prevented inflammation-induced impairment in foetal brain and lung tissues in lipopolysaccharide (LPS)-induced rodent models. However, it remains unclear whether $\mathrm{H}_{2}$ is capable of inhibiting preterm labour. The aim of the current study was therefore to investigate the effect of $\mathrm{H}_{2}$ on inflammation-induced preterm labour. Pregnant ICR (CD-1) mice were divided into three groups: Control, LPS and $\mathrm{H}_{2}$ water (HW) + LPS. In the control and LPS groups, vehicle and LPS, respectively, were intraperitoneally injected on embryonic day 15.5. In the HW + LPS group, HW was administered $24 \mathrm{~h}$ prior to LPS injection. The time from LPS administration to parturition was compared between the LPS and HW + LPS groups. Maternal uterus was collected $6 \mathrm{~h}$ after LPS injection and the transcript levels of pro-inflammatory cytokines, contractile-associated proteins (CAPs), matrix metalloproteinase-3 (Mmp3) and endothelin-1 (Etl)
\end{abstract}

Correspondence to: Dr Tomomi Kotani, Department of Obstetrics and Gynaecology, Nagoya University Graduate School of Medicine, 65 Tsurumai-cho, Showa-ku, Nagoya, Aichi 466-8550, Japan E-mail: itoto@med.nagoya-u.ac.jp

Abbreviations: ANOVA, analysis of variance; CAP, contractileassociated protein; Cox 2 (Ptgs2), cyclooxygenase-2 (prostaglandinendoperoxide synthase 2); Cx43 (Gja1), connexin-43 (gap junction protein alpha 1); E, embryonic day; Et1 (Edn1), endothelin-1; $\mathrm{HW}, \mathrm{H}_{2}$ water; Il6/8, interleukin-6/8; IS, intensity score; LPS, lipopolysaccharide; Mmp3, matrix metalloproteinase-3; NAC, $\mathrm{N}$-acetylcysteine; Oxtr, oxytocin receptor; PBS, phosphate-buffered saline; qPCR, quantitative polymerase chain reaction; Tnf, tumour necrosis factor

Key words: preterm labour, contractile-associated proteins, inflammation, progesterone were assessed by reverse transcription-quantitative polymerase chain reaction. The protein levels of cyclooxygenase-2 (Cox2) were also evaluated by immunohistochemistry. The time from LPS administration to parturition in the HW + LPS group was significantly increased compared with that in the LPS group (33.5 \pm 3.4 vs. $18.3 \pm 8.8 \mathrm{~h}$, respectively, $\mathrm{P}=0.020) . \mathrm{H}_{2}$ administration also resulted in significantly higher progesterone levels compared with LPS treatment alone $(\mathrm{P}=0.002)$. The transcript levels of pro-inflammatory cytokines, CAPs, Mmp3 and Etl in the uteri of the LPS group were significantly higher than those in the control group (all $\mathrm{P}<0.05$ ). In turn, all these levels with the exception of interleukin- 8 and Mmp3 were significantly lower in the HW + LPS group compared with those in the LPS group (all $\mathrm{P}<0.05$ ). The protein levels of Cox 2 in the LPS group were also significantly increased compared with those in the control $(\mathrm{P}<0.001)$ and HW + LPS $(\mathrm{P}=0.003)$ groups. These results suggest that inflammation-induced changes in the uterus may be ameliorated through maternal $\mathrm{H}_{2}$ administration. Preventive $\mathrm{H}_{2}$ administration may therefore represent an effective strategy for the suppression of inflammation during preterm labour.

\section{Introduction}

Preterm birth is a major cause of neonatal mortality and morbidity worldwide, and the leading cause of under-five mortality is complications of preterm birth (1). Improving preterm birth outcomes is therefore essential to reduce global under-five mortality. More specifically, intrauterine inflammation is a major cause of preterm labour and complications in preterm neonates (2). Ascending infection and invasion of microorganisms into the uterine cavity may lead to intrauterine inflammation, chorioamnionitis and foetal inflammatory response syndrome (3). Furthermore, these conditions are frequently associated with adverse long-term complications, which include pulmonary and neurological impairments (4). Thus, an approach to suppress intrauterine inflammation would reduce the risk of severe complications and avoid prematurity by extending the duration of pregnancy, both of which may improve the prognosis of neonates (5).

Previous reports by our group demonstrated that the maternal administration of molecular hydrogen $\left(\mathrm{H}_{2}\right)$, known 
for its anti-oxidative and anti-inflammatory effects (6), ameliorated damage in foetal pulmonary and brain tissue caused by intrauterine inflammation in lipopolysaccharide(LPS)-induced rodent models (7-9). There are more than 300 reports outlining the utility of $\mathrm{H}_{2}$ for the prevention or improvement of various diseases including inflammatory disease (6). $\mathrm{H}_{2}$ has been reported to be a novel antioxidant that selectively scavenges hydroxyl radicals and peroxynitrates (10), and to also have an anti-inflammatory effect through the modulation of signalling molecules (6). A recent report demonstrated that $\mathrm{H}_{2}$ suppressed $\mathrm{Ca}^{2+}$ signalling (11), while $\mathrm{Ca}^{2+}$ mobilization is important for uterine contraction and labour onset (12). From these findings, it may be speculated that $\mathrm{H}_{2}$ reduces proinflammatory cytokine production, which may lead to the suppression of uterine contractile-associated proteins (CAPs), $\mathrm{Ca}^{2+}$ signalling and preterm uterine contractions, ultimately resulting in the prevention of preterm delivery. However, the potential applications of $\mathrm{H}_{2}$ for the prevention of preterm birth remain to be elucidated.

To address this, the present study investigated the effects of $\mathrm{H}_{2}$ administration on uterine inflammation and aimed to determine whether $\mathrm{H}_{2}$ prevents LPS-induced preterm delivery. The effects of $\mathrm{H}_{2}$ on the expression of transcripts associated with inflammation, contraction and tissue remodeling were also evaluated. Identifying an association between the administration of $\mathrm{H}_{2}$ and preterm labour may lead to more effective strategies for preventing preterm labour.

\section{Materials and methods}

Reagents. LPS (Escherichia coli LPS, serotype O55:B5) was purchased from Sigma-Aldrich (Merck KGaA, Darmstadt, Germany). $\mathrm{H}_{2}$ water (HW) was gifted by Blue Mercury, Inc. (Tokyo, Japan) and was administered to pregnant mice, as described previously (13). Briefly, the HW was stored in aluminium bags at a $\mathrm{H}_{2}$ concentration of $>0.4 \mathrm{mM}$. $\mathrm{HW}$ for drinking was made available to mice from glass bottles with two ball bearings at the outlet to prevent $\mathrm{H}_{2}$ degassing. The concentration of $\mathrm{H}_{2}$ was maintained at $>0.2 \mathrm{mM}$, and $\mathrm{H}_{2}$ was replaced every $24 \mathrm{~h}$.

Animals and treatments. All protocols for animal experiments were approved by the Animal Experiment Committee of Nagoya University (approval no. 29154). A total of 38 pregnant ICR (CD-1) mice (51.01 \pm 0.86 g, 8-10 weeks old; Charles River Laboratories Japan, Kanagawa, Japan) were maintained under a 12-h light/dark cycle (lights on 09:00 a.m., lights off 09:00 p.m.). A total of 18 of the mice were randomly divided into the following three groups ( $n=6$ per group): Control, LPS and HW + LPS. In the LPS and control groups, mice were administered an intraperitoneal injection of $500 \mu \mathrm{g} \mathrm{LPS} / \mathrm{kg}$ body weight dissolved in phosphate-buffered saline (PBS), or the same volume of PBS, respectively, on embryonic day 15.5 (E15.5). In the HW + LPS group, the mice were administered HW as drinking water $24 \mathrm{~h}$ before LPS administration (E14.5) until sacrifice (E15.5). The mice drank approximately $200 \mathrm{ml} / \mathrm{kg}$ of regular water or HW per day, as previously reported (8). Samples of serum and uteri from the mice in each group were collected (storage at $-80^{\circ} \mathrm{C}$ for $<3$ months) following euthanasia $6 \mathrm{~h}$ after the intraperitoneal injection of
PBS or LPS (E15.5). To evaluate the period between injection of LPS and delivery, the 20 remaining mice allocated to LPS and HW + LPS groups ( $=10$ per group) were checked for parturition every $1 \mathrm{~h}$ after LPS administration. Parturition was not examined in a control group, as this is presumed to occur at approximately E18-19, as previously reported (14). The parturition time was determined as the time at which mice had delivered all pups.

Measurement of progesterone. The concentration of progesterone in maternal serum (E15.5) was evaluated with a commercially available ELISA kit (ADI-900-011; Enzo Life Sciences, Inc., Farmingdale, NY, USA), according to the manufacturer's instructions. The absorbance was read at $405 \mathrm{~nm}$ with a microplate reader (ELx808; BioTek Instruments, Inc., Winooski, VT, USA).

Reverse transcription-quantitative polymerase chain reaction ( $q P C R)$. Total RNA was extracted from uteri samples using an RNeasy mini kit (Qiagen K.K., Tokyo, Japan), and was reverse transcribed using ReverTra Ace (Toyobo Co., Ltd., Osaka, Japan), according to the manufacturer's instructions. qPCR was performed on a Thermal Cycler Dice Real Time System TP800 (Takara Bio, Inc., Otsu, Japan) using SYBR Premix Ex Taq II (Takara Bio, Inc.) with use of a crossing point method for quantification (15). The cycling profile was as follows: Initial denaturation at $95^{\circ} \mathrm{C}$ for $30 \mathrm{sec}$; denaturation at $95^{\circ} \mathrm{C}$ for $5 \mathrm{sec}$ and annealing at $60^{\circ} \mathrm{C}$ for $30 \mathrm{sec}$ (40 cycles); and dissociation at $95^{\circ} \mathrm{C}$ for $5 \mathrm{sec}, 55^{\circ} \mathrm{C}$ for $10 \mathrm{sec}$ and $72^{\circ} \mathrm{C}$ for $20 \mathrm{sec}$. The expressions of target genes were normalized according to $\beta$-actin expression. The primer sequences used were as follows: For mouse $\beta$-actin forward, 5'-CGTGGGCCGCCCTAGG CACCA-3' and reverse, 5'-ACACGCAGCTCATTGTA-3'; for mouse interleukin-6 (Il6) forward, 5'-ACAACCACGGCCT TCCCTAC-3' and reverse, 5'-TCCACGATTTCCCAGAGA ACA-3', as previously reported (9); for mouse tumour necrosis factor (Tnf) forward, 5'-GTAGCCCACGTCGTAGCAAAC-3' and reverse, 5'-CTGGCACCACTAGTTGGTTGTC-3'; for mouse $I l 8$ forward, 5'-GCCCAGACAGAAGTCATAGAA-3' and reverse, 5'-AGGCTCCTCCTTTCCAGGTC-3'; for mouse cyclooxygenase-2 [Cox2; also known as prostaglandin-endoperoxide synthase 2 (Ptgs2)] forward, 5'-TGCCCAGCAC TTCACCCATCA-3' and reverse, 5'-AGTCCACTCCATGG CCCAGTCC-3'; for mouse connexin-43 [Cx43; also known as gap junction protein alpha 1 (Gjal)] forward, 5'-TAAGTGA AAGAGAGGTGCCCAGA-3' and reverse, 5'-GTGGAGTAG GCTTGGACCTTG-3'; for mouse oxytocin receptor (Oxtr) forward, 5'-TCATCGTGTGCTGGACGCCT-3' and reverse, 5'-TGTTGAGGCTGGCCAAGAGCAT-3'; for mouse matrix metalloproteinase-3 (Mmp3) forward, 5'-GCTGTCTTTGAA GCATTTGGGTT-3' and reverse, 5'-ACACAGGATGCCTTC CTTGGAT-3'; and for mouse endothelin-1 (Et1; also known as $E d n 1$ ) forward, 5'-ACTTGCTGAGGACCGCGCTG-3' and reverse, 5'-GCTCCGGTGCTGAGTTCGGC-3'. All primers were purchased from Nippon Gene Co., Ltd. (Tokyo, Japan).

Immunohistochemistry. The uteri samples ( $\mathrm{n}=6$ per group) were fixed with $4 \%$ paraformaldehyde phosphate buffer solution (163-20145; Wako Pure Chemical Industries, Ltd., Osaka, Japan) at $4^{\circ} \mathrm{C}$ for $48 \mathrm{~h}$, and maintained in $70 \%$ ethanol at $4^{\circ} \mathrm{C}$ 
for $<3$ months, then embedded in paraffin. For heat-induced epitope retrieval, deparaffinised sections at a thickness of $4 \mu \mathrm{m}$ were placed in $1 \mathrm{mM}$ citrate buffer ( $\mathrm{pH} \mathrm{6.0)}$ ), and heated at $90^{\circ} \mathrm{C}$ and $750 \mathrm{~W}$ using an $\mathrm{H} 2500$ microwave oven for 20 min. Staining was performed using a Histofine SAB-PO (R) kit (Nichirei Biosciences, Inc., Tokyo, Japan) with diaminobenzidine as the chromogen, based on the manufacturer's instructions. Rabbit polyclonal anti-Cox2 (ab52237; Abcam, Cambridge, UK) was used at a 1:400 dilution as the primary antibody at $4^{\circ} \mathrm{C}$ overnight. Finally, the slides were counterstained with Meyer's haematoxylin at room temperature for $30 \mathrm{sec}$. For the quantification of immunostaining, the intensity was evaluated as intensity score (IS), as previously reported (9). Two independent examiners determined the IS from three segments per slide at $x 400$ magnification with an Axio Imager A1 (Carl Zeiss Microscopy Co., Ltd., Tokyo, Japan) using a 4-point system: 0, 1, 2 and 3 (for no, light, medium and dark staining, respectively); the mean score from the two examiners was used.

Statistical analysis. The data are presented as the means \pm standard error of the mean. For comparisons between two groups including comparisons of times to parturition and progesterone levels, data were analysed by performing a Student's t-test or Mann-Whitney U test, according to whether distributions were normal or non-normal, respectively. For comparison of the rate of preterm birth, a Fisher's exact test was used. To compare three groups, one-way analysis of variance (ANOVA) with Kruskal-Wallis post hoc analysis was used, and P-values were also calculated between two of the three groups, using a Student's t-test or Mann-Whitney U test, as above. When the distribution of data was normal, P-values were calculated by performing one-way ANOVA with a Tukey's honest significant difference or Games-Howell test, according to whether variance was equal or unequal, respectively. Statistical analyses were performed using the SPSS 24 software package (IBM Corp., Armonk, NY, USA). Differences between values with $\mathrm{P}<0.05$ were considered statistically significant.

\section{Results}

Effect of $\mathrm{H}_{2}$ on preterm labour. The period between injection of LPS and delivery was significantly increased in the HW + LPS group compared with the LPS alone group (33.5 \pm 3.4 vs. $18.3 \pm 8.8$ h, respectively, $\mathrm{P}=0.020$; Fig. $1 \mathrm{~A}$ ). However, the rate of preterm birth in the LPS group was not significantly different from that of the HW + LPS group [number of dams that delivered pups within $24 \mathrm{~h}$ after LPS administration/total number $=9 / 10(90 \%)$ vs. $6 / 10(60 \%)$, respectively, $\mathrm{P}=0.303$; data not shown]. The concentration of progesterone in the maternal serum of the HW + LPS group was significantly higher than that in the LPS group $(7.30 \pm 0.04$ vs. $7.14 \pm 0.02 \times 10^{-2} \mathrm{pg} / \mathrm{ml}$, respectively, $\mathrm{P}=0.002$; Fig. 1B).

$\mathrm{H}_{2}$ pretreatment reduces LPS-induced proinflammatory cytokine levels in the uterus. As depicted in Fig. 2, the expression of Tnf, Il6, and Il8 mRNA in the uteri of the LPS group was significantly increased compared with that in the control group $(\mathrm{P}=0.006, \mathrm{P}=0.004$ and $\mathrm{P}=0.004$, respectively). Maternal HW administration inhibited the expression of $\operatorname{Tnf}$ to levels
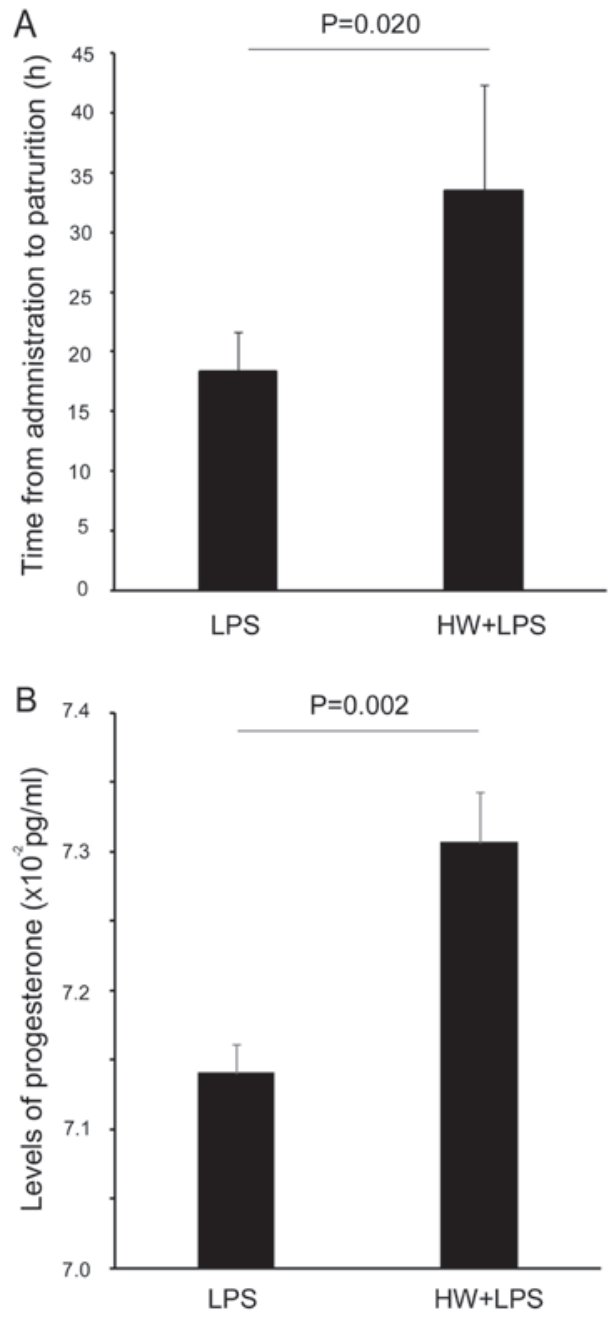

Figure 1. Effect of $\mathrm{H}_{2}$ on LPS-induced preterm labour. (A) Time from intraperitoneal LPS administration to parturition (h) in LPS vs. HW + LPS groups. The results are expressed as the means \pm standard error of the mean $(n=10$ per group). The time from intraperitoneal LPS administration to parturition was significantly increased in the HW + LPS group. The indicated P-value was calculated using the Mann-Whitney U test. (B) Levels of progesterone $\left(\mathrm{x} 10^{-2} \mathrm{pg} / \mathrm{ml}\right)$ in LPS vs. HW + LPS groups. The results are expressed as the means \pm standard error of the mean ( $n=6$ per group). The progesterone level in the maternal serum of the HW + LPS group was significantly increased compared with that in the LPS group. The P-value was calculated according to the Student's t-test. LPS, lipopolysaccharide; $\mathrm{HW}, \mathrm{H}_{2}$ water.

equivalent to those observed in the control group $(\mathrm{P}=0.337$; Fig. 2A). The expression of $I l 6$ in the uteri of the HW + LPS group was significantly lower than that in the LPS group $(\mathrm{P}=0.037)$, but remained higher than that in the control group ( $\mathrm{P}=0.016$; Fig. 2B). The expression of $I l 8 \mathrm{mRNA}$ in the uteri of the HW + LPS group was not significantly lower compared to that in the LPS group ( $\mathrm{P}=0.749$; Fig. $2 \mathrm{C})$.

$\mathrm{H}_{2}$ pretreatment downregulates LPS-induced CAPs in the uterus. As indicated in Fig. 3, the expression of several contractile-associated proteins (CAPs) transcripts including Cox2 (Ptgs2), Cx43 (Gjal) and Oxtr was significantly higher in the uteri of the LPS group compared with that in the control group ( $\mathrm{P}=0.004, \mathrm{P}=0.004$ and $\mathrm{P}=0.006$, respectively). Conversely, their levels were lower in the uteri of the HW + LPS group compared with those in the LPS group (all $\mathrm{P}=0.004$ ). 
A

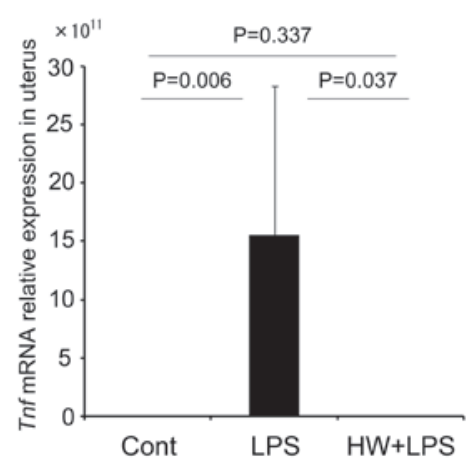

B

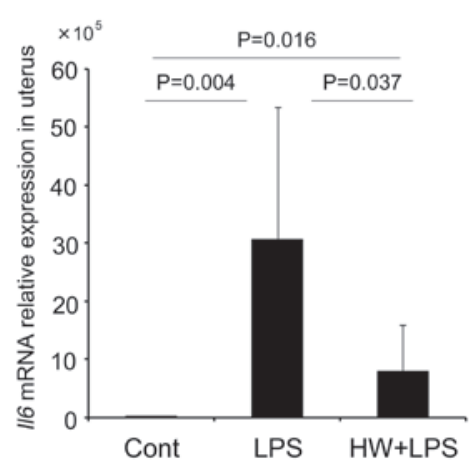

C

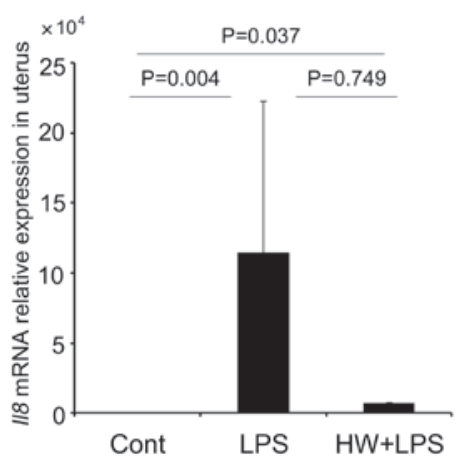

Figure 2. Levels of $T n f, I l 6$ and $I l 8$ mRNA measured by reverse transcription-quantitative polymerase chain reaction. The data were normalized to the expression of $\beta$-actin and are expressed as the means \pm standard error of the mean ( $\mathrm{n}=6$ per group). (A) $T n f$, (B) $I l 6$ and (C) $I l 8$ mRNA levels in the uterus of the LPS group were significantly higher compared with those in the control group; whereas, the levels in the HW + LPS group were lower compared with those in the LPS group. All indicated P-values were calculated using the Mann-Whitney $\mathrm{U}$ test. The data among the three groups were also statistically analysed by the Kruskal-Wallis test, and $\mathrm{P}$-values for $T n f, I l 6$, and $I l 8$ were $\mathrm{P}=0.013, \mathrm{P}=0.002$ and $\mathrm{P}=0.015$, respectively. Tnf, tumour necrosis factor; $\mathrm{Il6} / 8$, interleukin-6/8; LPS, lipopolysaccharide; $\mathrm{HW}, \mathrm{H}_{2}$ water.
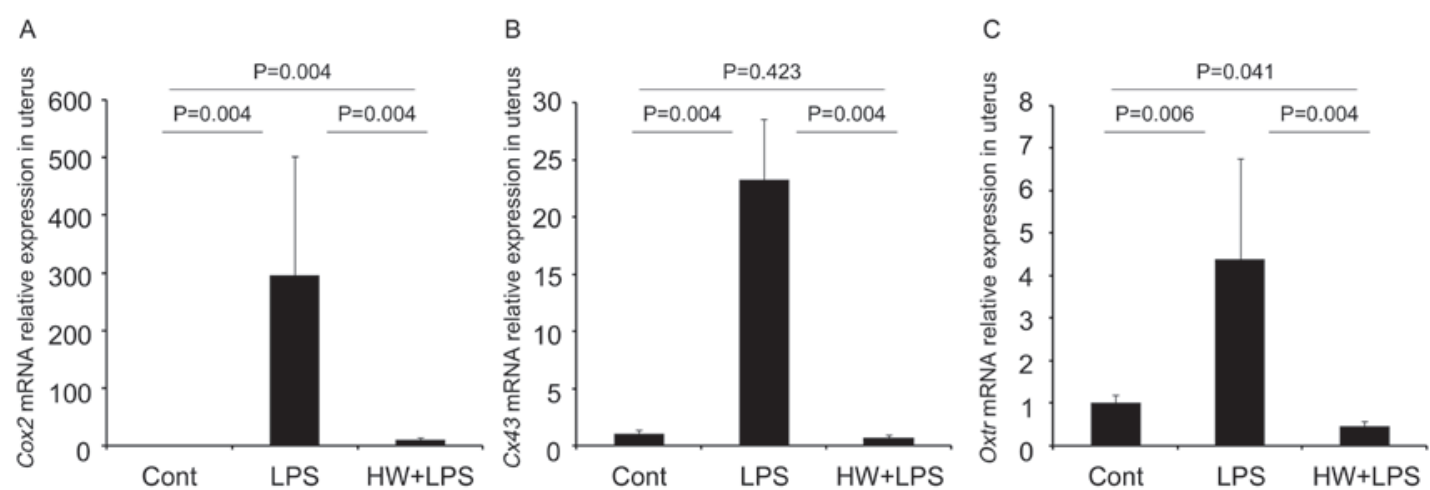

Figure 3. mRNA levels of contractile-associated proteins measured by reverse transcription-quantitative polymerase chain reaction. The data were normalized to the expression of $\beta$-actin. The results are expressed as the means \pm standard error of the mean ( $\mathrm{n}=6$ per group). The mRNA levels of (A) Cox2, (B) Cx43 and (C) Oxtr were significantly increased in the uteri of the LPS group compared with those in the control group; whereas, levels in the HW + LPS group were significantly lower compared with those in the LPS group. All indicated P-values were calculated using the Mann-Whitney U test, with the exception of $O x t r$ (control vs. HW + LPS), which was calculated using the Student's t-test. The data among the three groups were also statically analysed by the Kruskal-Wallis test, and the $\mathrm{P}$-values for Cox2, Cx43 and Oxtr were $\mathrm{P}=0.001, \mathrm{P}=0.003$, and $\mathrm{P}=0.002$, respectively. Cox 2, cyclooxygenase-2; Cx43, connexin-43; Oxtr, oxytocin receptor; LPS, lipopolysaccharide; $\mathrm{HW}, \mathrm{H}_{2}$ water.

Furthermore, the level of $C x 43$ mRNA in the HW + LPS group did not differ significantly different from that in the control group ( $\mathrm{P}=0.423 ;$ Fig. 3B). The expression of Cox2 in the uteri of the HW + LPS group remained higher than that in the control group ( $\mathrm{P}=0.004$; Fig. 3A). Meanwhile, Oxtr mRNA expression in the uteri of the HW + LPS group was lower than that in the control group ( $\mathrm{P}=0.041$; Fig. $3 \mathrm{C})$. The protein expression of Cox2 (Ptgs2) was significantly higher in the uteri of the LPS group compared with that in the control group $(\mathrm{P}<0.001$; Fig. 4A and $\mathrm{B})$, while the expression level was lower in the uteri of the $\mathrm{HW}+$ LPS group ( $\mathrm{P}=0.003$ vs. LPS group; Fig. $4 \mathrm{~A}$ and $\mathrm{B})$. In addition, the expression of Cox 2 in the HW + LPS group did not differ significantly different from that in the control group ( $\mathrm{P}=0.167$; Fig. 4A and B). Mmp3 expression was also significantly higher in the LPS group compared with that in the control group ( $\mathrm{P}=0.037)$, and was lower in the HW + LPS group compared with that in the LPS group, but the difference was not significant ( $\mathrm{P}=0.055$; Fig. 5). Although notably, the expression of $M m p 3$ in the HW + LPS group did not differ significantly different from that in the control group ( $\mathrm{P}=0.873$; Fig. 5). Etl
(Edn1) expression was significantly higher in the uteri of the LPS group relative to that in the control group ( $\mathrm{P}=0.001)$, whereas levels in the HW + LPS group were significantly lower compared with those in the LPS group ( $\mathrm{P}=0.019$; Fig. 6). Et1 mRNA levels did not differ significantly different between the control and HW + LPS groups ( $\mathrm{P}=0.177$; Fig. 6).

\section{Discussion}

Previously, studies by our group have identified that $\mathrm{H}_{2}$ had the potential to prevent foetal brain and lung impairment due to maternal inflammation. Notably, $\mathrm{H}_{2}$ was demonstrated to exert anti-oxidative and anti-inflammatory effects in foetal organs following intraperitoneal injection of LPS in rodent models (7-9). It is established that preterm labour is associated with inflammation, but the associated mechanism is not fully understood. Pro-inflammatory cytokines in the uterus, including IL6, increase CAPs, which initiates the onset of labour (16). To our knowledge, the present study is the first to report that $\mathrm{H}_{2}$ may also significantly prevent uterine 
A

Control

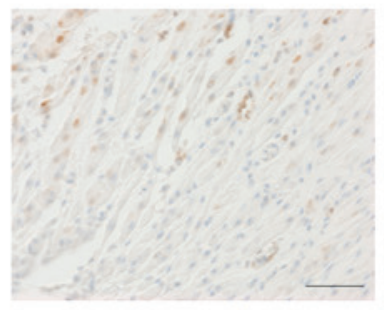

LPS

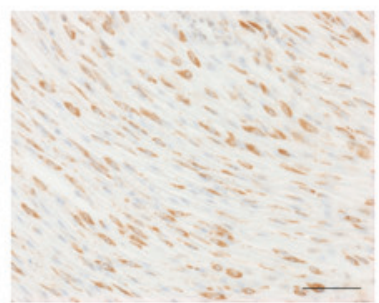

HW+LPS

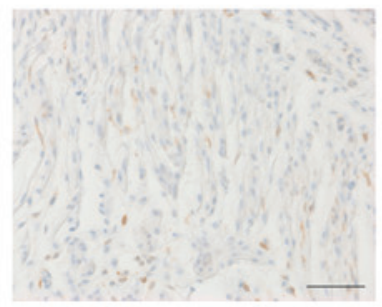

B

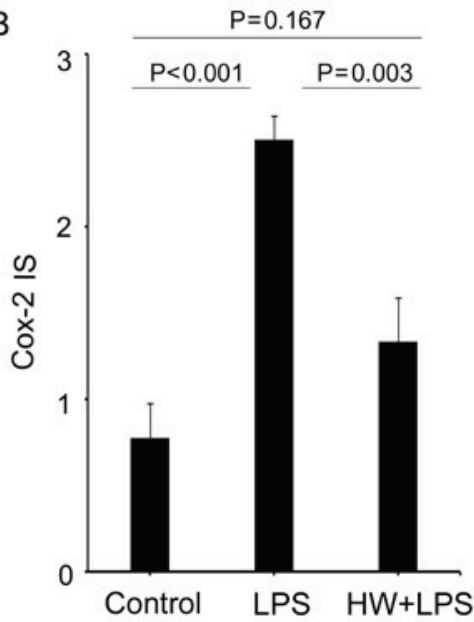

Figure 4. Protein expression of Cox 2 assessed by immunohistochemistry. (A) Immunohistochemical staining for Cox 2 in the uteri. Magnification, $\mathrm{x} 400$, scale bars, $5 \mu \mathrm{m}$. Cox 2 staining in the uteri of the LPS group was stronger than that in the control group (n=6 per group). (B) Cox 2 staining was scored as IS for quantitative evaluation. The IS in the LPS group was significantly higher than that in the control group, whereas that in the HW + LPS group was significantly lower than that in the LPS group. The P-values were calculated using the Tukey's honest significant difference test. Cox2, cyclooxygenase-2; LPS, lipopolysaccharide; $\mathrm{HW}, \mathrm{H}_{2}$ water; IS, intensity score.

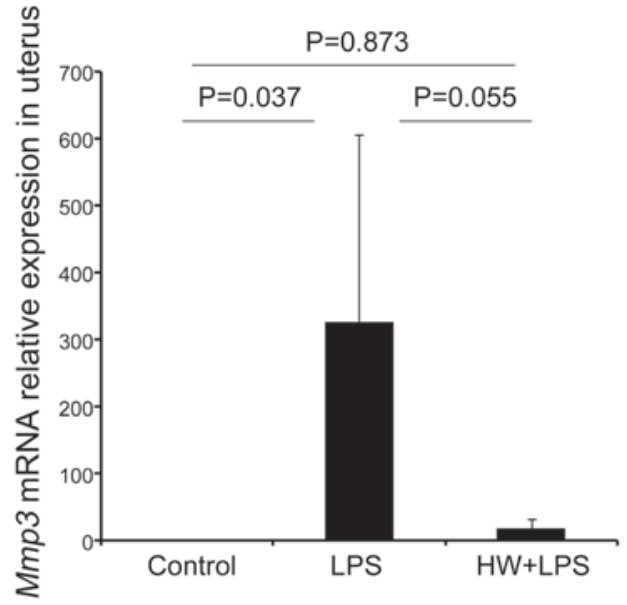

Figure 5. Levels of Mmp3 mRNA measured by reverse transcription-quantitative polymerase chain reaction. Data were normalized to the expression of $\beta$-actin. The results are expressed as the means \pm standard error of the mean ( $\mathrm{n}=6$ per group). Mmp3 expression in the uteri of the LPS group was significantly higher than that in the control group; whereas, levels were lower in the HW + LPS group compared with those in the LPS group. The indicated P-values were calculated using the Mann-Whitney U test. The data among the three groups were also statistically analysed by the Kruskal-Wallis test, and the $\mathrm{P}$-value for $\mathrm{Mmp3}$ was $\mathrm{P}=0.064$. Mmp3, matrix metalloproteinase-3; LPS, lipopolysaccharide; $\mathrm{HW}, \mathrm{H}_{2}$ water.

inflammation, potentially extending the duration of pregnancy in a murine model of LPS-induced preterm birth.

This effect may be partially dependent on a significant increase in progesterone levels. However, while $\mathrm{H}_{2}$ pretreatment did not reduce the rates of LPS-induced preterm birth in the present study, it did extend the duration of pregnancy. The reason for this effect is unknown. However, it is notable that the expression of certain transcripts including Tnf, Cx43 (Gjal), Oxtr and Etl (Ednl) was reduced to levels equivalent to those observed in the control group, despite no observable effects on Il 8 and $M m p 3$, by $\mathrm{H}_{2}$ administration. IL8 is expressed only by the myometrium during active labour, whereas IL6 is present

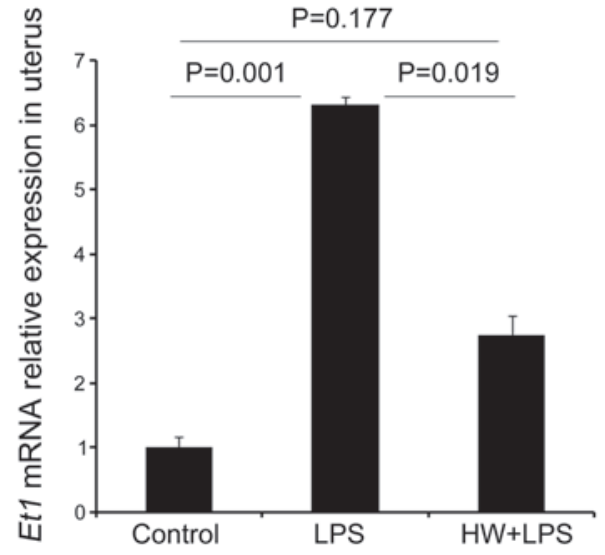

Figure 6. Levels of Et1 mRNA measured by reverse transcription-quantitative polymerase chain reaction. Data were normalized to the expression of $\beta$-actin. The results are expressed as the means \pm standard error of the mean (n=6 per group). Et1 expression in the uteri of the LPS group was significantly higher than that in the control group, whereas in the HW + LPS group, levels were significantly reduced compared with those in the LPS group. The P-values were calculated using the Games-Howell test. Et1, endothelin-1; LPS, lipopolysaccharide; $\mathrm{HW}, \mathrm{H}_{2}$ water.

in the preterm myometrium prior to and during labour (17), and no significant effect on $\mathrm{Il} 8$ expression by $\mathrm{H}_{2}$ pretreatment may lead to a continuation of active labour, leading to preterm birth while extending the period of delivery.

Progesterone, a steroid hormone that is essential for the maintenance of pregnancy, has been demonstrated to decrease preterm birth risk in high-risk populations, including in women with short cervices (18). However, while the underlying molecular mechanisms are not fully understood, maternal plasma progesterone has been reported to be lower in LPS-induced preterm birth models (19), and unaltered by probiotics (20). $\mathrm{H}_{2}$ may therefore be superior in this regard, as a previous study reported that progesterone exerted anti-inflammatory effects at the maternal-foetal interface and increased the proportion of decidual regulatory T cells (21). These findings suggest that 
$\mathrm{H}_{2}$ may also exert an anti-inflammatory effect in combination with increasing maternal serum progesterone.

Additionally, CAPs are an important family of proteins that are involved in the onset of labour. For example, the expression of certain CAPs, including Cox 2 (Ptgs2), Cx43 (Gja1) and Oxtr, has been reported to be elevated in the murine uterus at preterm birth to an equivalent level to that observed at term (22). More specifically, Oxtr has been reported to be increased in the human uterus following the onset of labour, both at term and preterm (23). Furthermore, an Oxtr antagonist, atosiban, elicited similar outcomes to nifedipine during tocolysis (24). Increased Cox 2 expression has also been detected in the human myometrium during preterm labour (25). Finally, Cx43 protein expression has been identified to increase at term and with the onset of labour in humans (26), although the expression of $\mathrm{Cx} 43$ during preterm labour remains poorly understood in humans. Collectively, these findings indicate that a reduction in the levels of CAPs in the uterus may inhibit preterm labour. In the present study, $\mathrm{H}_{2}$ pretreatment suppressed the levels of LPS-induced CAPs in the uterus. N-acetylcysteine (NAC), which may serve as an antioxidant alike $\mathrm{H}_{2}$, has also been reported to decrease the ratio of preterm births in an animal model of LPS-induced preterm birth (27), although NAC did not reduce $\mathrm{Cx} 43$ or Oxtr expression (26).

MMP3 serves an important role in the tissue remodelling that occurs in the human myometrium during labour. Accordingly, levels of MMP3 have been reported to be increased at labour (28). Furthermore, LPS treatment increased MMP3 levels in human cervical smooth-muscle cells (29) and rabbit cervical tissues (30). In the present study, maternal administration of $\mathrm{H}_{2}$ did not significantly decrease LPS-induced Mmp3 mRNA expression levels. Thus, the rates of preterm birth may not have been decreased by $\mathrm{H}_{2}$ pretreatment because the preventive effect of $\mathrm{H}_{2}$ on cervical remodelling in the uterus was incomplete.

Et1 (Edn1) also serves a pathological role in inflammation-induced preterm labour (31) and is increased in the amniotic fluid of women with infection-induced preterm labour (32). Wang et al (33) demonstrated that the levels of ET1 were increased in the gestational tissues of a murine model of LPS-induced preterm birth, supporting the results of the present study. These authors additionally observed that RNA silencing of endothelin-converting enzyme-1 and a selective endothelin receptor A antagonist (BQ-123) were effective at preventing LPS-induced preterm labour, and decreased the percentages of preterm births to approximately 14 and $16 \%$, respectively; while in the intraperitoneally injected LPS group, 70-90\% of pregnant mice delivered pups preterm (33). In the present study, Etl (Ednl) levels also decreased significantly following maternal $\mathrm{H}_{2}$ administration. However, this did not prevent preterm birth in the present study. The inconsistency in the prevention of preterm birth may be due to differences in the mouse strain used or the dose of LPS administered.

The present study suggests that, while $\mathrm{H}_{2}$ pretreatment may elicit effects on uterine molecules involved in preterm labour, it only has weak effects on the prevention of preterm birth. Our previous reports demonstrated that may $\mathrm{H}_{2}$ prevent foetal inflammation and oxidative damage (7-9), and compared to the effects of $\mathrm{H}_{2}$ on foetal damage, its effects on preterm labour were limited. These findings suggest that $\mathrm{H}_{2}$ pretreatment may be appropriate for preventing foetal injury from maternal inflammation at preterm labour, but has low potential to inhibit preterm birth. However, as $\mathrm{H}_{2}$ significantly altered the expression of a number of important molecules expressed in the uterus, it may be effective for the prevention of mild or chronic inflammation-related preterm birth. The model used in the present study mimics an acute inflammation-induced preterm birth, and resulted in preterm birth at $18.3 \pm 8.8 \mathrm{~h}$ after intraperitoneal LPS administration. A recent study reported that chronic inflammation also serves a role in preterm labour (34) and thus further investigation is required to fully understand the effects of $\mathrm{H}_{2}$ on chronic inflammation-induced preterm birth.

However, the present study included a limitation in that $\mathrm{H}_{2}$ was administered prior to LPS injection as a preventive protocol. Most anti-oxidants and anti-inflammatory agents are known to be effective when administered prior to injury. In an unpublished preliminary study by our group, $\mathrm{H}_{2}$ exerted only a minimal effect on the foetus when the timing of administration was delayed to after the onset of injury. This finding highlights the importance of the early detection of intrauterine inflammation. In addition, the mechanism associated with the effect of $\mathrm{H}_{2}$, including signalling pathways in the uterine wall remain unknown, and further investigations are required.

In conclusion, prolonging pregnancy in response to maternal administration of $\mathrm{H}_{2}$ for preterm labour in a model of acute inflammation was minimal. However, the anti-inflammatory effects and the reduction in the levels of expression of several CAPs were observed. Maternal administration of $\mathrm{H}_{2}$ may therefore be ineffective in preventing preterm birth associated with acute inflammation, although the effects of maternal $\mathrm{H}_{2}$ administration on chronic inflammation-induced preterm birth should be investigated further.

\section{Acknowledgements}

The authors are thankful to Blue Mercury, Inc., Tokyo, Japan for contributing the $\mathrm{H}_{2}$ water.

\section{Funding}

The current study was supported by the Japan Society for the Promotion of Science Grants-in-Aid for Scientific Research program (KAKENHI; grant nos. 15H06282 and 26462484).

\section{Availability of data and materials}

The analyzed data sets generated during the study are available from the corresponding author on reasonable request.

\section{Authors' contributions}

TN performed experiments and data analyses, and wrote the manuscript. TK designed the study, analysed and interpreted data and wrote the manuscript. KI, YI, TU, HT and HL performed experiments and data analyses. AI, ST and FK aided with data interpretation, and edited the manuscript. The 
final version of the manuscript has been read and approved by all authors.

\section{Ethics approval and consent to participate}

All protocols for animal experiments were approved by the Animal Experiment Committee of Nagoya University (approval no. 29154).

\section{Consent for publication}

Not applicable.

\section{Competing interests}

The authors declare that they have no competing interests.

\section{References}

1. Liu L, Oza S, Hogan D, Chu Y, Perin J, Zhu J, Lawn JE, Cousens S, Mathers $\mathrm{C}$ and Black RE: Global, regional, and national causes of under-5 mortality in 2000-15: An updated systematic analysis with implications for the sustainable development goals. Lancet 388 3027-3035, 2016

2. Boyle AK, Rinaldi SF, Norman JE and Stock SJ: Preterm birth: Inflammation, fetal injury and treatment strategies. J Reprod Immunol 119: 62-66, 2017

3. Kim CJ, Romero R, Chaemsaithong P, Chaiyasit N, Yoon BH and Kim YM: Acute chorioamnionitis and funisitis: Definition, pathologic features, and clinical significance. Am J Obstet Gynecol 213 (Suppl): S29-S52, 2015.

4. Chau V, McFadden DE, Poskitt KJ and Miller SP: Chorioamnionitis in the pathogenesis of brain injury in preterm infants. Clin Perinatol 41: 83-103, 2014.

5. Keelan JA and Newnham JP: Editorial: Advances in the prevention and treatment of inflammation-associated preterm birth. Front Immunol 7: 264, 2016.

6. Ichihara M, Sobue S, Ito M, Ito $M$, Hirayama $M$ and Ohno $K$ : Beneficial biological effects and the underlying mechanisms of molecular hydrogen - comprehensive review of 321 original articles. Med Gas Res 5: 12, 2015.

7. Hattori Y, Kotani T, Tsuda H, Mano Y, Tu L, Li H, Hirako S, Ushida T, Imai K, Nakano T, et al: Maternal molecular hydrogen treatment attenuates lipopolysaccharide-induced rat fetal lung injury. Free Radic Res 49: 1026-1037, 2015.

8. Imai K, Kotani T, Tsuda H, Mano Y, Nakano T, Ushida T, Li H, Miki R, Sumigama S, Iwase A, et al: Neuroprotective potential of molecular hydrogen against perinatal brain injury via suppression of activated microglia. Free Radic Biol Med 91: 154-163, 2016.

9. Nakano T, Kotani T, Mano Y, Tsuda H, Imai K, Ushida T, Li H, Miki R, Sumigama S, Sato Y, et al: Maternal molecular hydrogen administration on lipopolysaccharide-induced mouse fetal brain injury. J Clin Biochem Nutr 57: 178-182, 2015.

10. Ohsawa I, Ishikawa M, Takahashi K, Watanabe M, Nishimaki K, Yamagata K, Katsura K, Katayama Y, Asoh S and Ohta S: Hydrogen acts as a therapeutic antioxidant by selectively reducing cytotoxic oxygen radicals. Nat Med 13: 688-694, 2007.

11. Iuchi K, Imoto A, Kamimura N, Nishimaki K, Ichimiya H, Yokota $\mathrm{T}$ and Ohta S: Molecular hydrogen regulates gene expression by modifying the free radical chain reaction-dependent generation of oxidized phospholipid mediators. Sci Rep 6: 18971, 2016.

12. Herington JL, Swale DR, Brown N, Shelton EL, Choi H, Williams CH, Hong CC, Paria BC, Denton JS and Reese J: High-throughput screening of myometrial calcium-mobilization to identify modulators of uterine contractility. PLoS One 10: e0143243, 2015.

13. Nakashima-Kamimura N, Mori T, Ohsawa I, Asoh S and Ohta S: Molecular hydrogen alleviates nephrotoxicity induced by an anti-cancer drug cisplatin without compromising anti-tumor activity in mice. Cancer Chemother Pharmacol 64: 753-761, 2009.

14. Rinaldi SF, Catalano RD, Wade J, Rossi AG and Norman JE: Decidual neutrophil infiltration is not required for preterm birth in a mouse model of infection-induced preterm labor. J Immunol 192: 2315-2325, 2014.
15. Larionov A, Krause A and Miller W: A standard curve based method for relative real time PCR data processing. BMC Bioinformatics 6: 62, 2005.

16. Sivarajasingam SP, Imami N and Johnson MR: Myometrial cytokines and their role in the onset of labour. J Endocrinol 231: R101-R119, 2016.

17. Sehringer B, Schäfer WR, Wetzka B, Deppert WR, BrunnerSpahr R, Benedek E and Zahradnik HP: Formation of proinflammatory cytokines in human term myometrium is stimulated by lipopolysaccharide but not by corticotropin-releasing hormone. J Clin Endocrinol Metab 85: 4859-4865, 2000.

18. Romero R, Nicolaides KH, Conde-Agudelo A, O'Brien JM, Cetingoz E, Da Fonseca E, Creasy GW and Hassan SS: Vaginal progesterone decreases preterm birth $\leq 34$ weeks of gestation in women with a singleton pregnancy and a short cervix: An updated meta-analysis including data from the OPPTIMUM study. Ultrasound Obstet Gynecol 48: 308-317, 2016.

19. Aisemberg J, Vercelli CA, Bariani MV, Billi SC, Wolfson ML and Franchi AM: Progesterone is essential for protecting against LPS-induced pregnancy loss. LIF as a potential mediator of the anti-inflammatory effect of progesterone. PLoS One 8: e56161, 2013

20. Yang S, Li W, Challis JR, Reid G, Kim SO and Bocking AD: Probiotic Lactobacillus rhamnosus GR-1 supernatant prevents lipopolysaccharide-induced preterm birth and reduces inflammation in pregnant CD-1 mice. Am J Obstet Gynecol 211: 44.e1-44.e12, 2014

21. Filipovich Y, Klein J, Zhou Y and Hirsch E: Maternal and fetal roles in bacterially induced preterm labor in the mouse. Am J Obstet Gynecol 214: 386.e1-386.e9, 2016.

22. Cook JL, Zaragoza DB, Sung DH and Olson DM: Expression of myometrial activation and stimulation genes in a mouse model of preterm labor: Myometrial activation, stimulation, and preterm labor. Endocrinology 141: 1718-1728, 2000.

23. Fuchs AR, Fuchs F, Husslein P and Soloff MS: Oxytocin receptors in the human uterus during pregnancy and parturition. Am J Obstet Gynecol 150: 734-741, 1984.

24. van Vliet EOG, Nijman TAJ, Schuit E, Heida KY, Opmeer BC, Kok M, Gyselaers W, Porath MM, Woiski M, Bax CJ, et al: Nifedipine versus atosiban for threatened preterm birth (APOSTEL III): A multicentre, randomised controlled trial. Lancet 387: 2117-2124, 2016.

25. Mitsuya K, Singh N, Sooranna SR, Johnson MR and Myatt L: Epigenetics of human myometrium: DNA methylation of genes encoding contraction-associated proteins in term and preterm labor. Biol Reprod 90: 98, 2014.

26. Chow L and Lye SJ: Expression of the gap junction protein connexin-43 is increased in the human myometrium toward term and with the onset of labor. Am J Obstet Gynecol 170: 788-795, 1994.

27. Chang EY, Zhang J, Sullivan S, Newman R and Singh I: N-acetylcysteine attenuates the maternal and fetal proinflammatory response to intrauterine LPS injection in an animal model for preterm birth and brain injury. J Matern Fetal Neonatal Med 24: 732-740, 2011.

28. O'Brien M, O'Shaughnessy D, Ahamide E, Morrison JJ and Smith TJ: Differential expression of the metalloproteinase MMP3 and the alpha5 integrin subunit in human myometrium at labour. Mol Hum Reprod 13: 655-661, 2007.

29. Watari M, Watari H, Nachamkin I and Strauss JF III: Lipopolysaccharide induces expression of genes encoding pro-inflammatory cytokines and the elastin-degrading enzyme, cathepsin $\mathrm{S}$, in human cervical smooth-muscle cells. J Soc Gynecol Investig 7: 190-198, 2000

30. Nakayama K, Otsuki K, Yakuwa K, Hasegawa A, Sawada M, Mitsukawa K, Chiba H, Nagatsuka M and Okai T: Recombinant human lactoferrin inhibits matrix metalloproteinase (MMP-2, MMP-3, and MMP-9) activity in a rabbit preterm delivery model. J Obstet Gynaecol Res 34: 931-934, 2008.

31. Breuiller-Fouché M, Morinière C, Dallot E, Oger S, Rebourcet R, Cabrol D and Leroy MJ: Regulation of the endothelin/endothelin receptor system by interleukin-1\{beta\} in human myometrial cells. Endocrinology 146: 4878-4886, 2005.

32. Romero R, Avila C, Edwin SS and Mitchell MD: Endothelin-1,2 levels are increased in the amniotic fluid of women with preterm labor and microbial invasion of the amniotic cavity. Am J Obstet Gynecol 166: 95-99, 1992.

33. Wang W, Yen H, Chen CH, Soni R, Jasani N, Sylvestre G and Reznik SE: The endothelin-converting enzyme-1/endothelin-1 pathway plays a critical role in inflammation-associated premature delivery in a mouse model. Am J Pathol 173: 1077-1084, 2008.

34. Kim CJ, Romero R, Chaemsaithong P and Kim JS: Chronic inflammation of the placenta: Definition, classification, pathogenesis, and clinical significance. Am J Obstet Gynecol 213 S53-S69, 2015. 